Check for updates

Cite this: RSC Adv., 2018, 8, 2271

Received 21st October 2017

Accepted 27th December 2017

DOI: 10.1039/c7ra11628d

rsc.li/rsc-advances

\section{Interaction between functionalized graphene and sulfur compounds in a lithium-sulfur battery - a density functional theory investigation $\uparrow$}

\author{
Kimal Chandula Wasalathilake, (D) Md Roknuzzaman, (D) Kostya (Ken) Ostrikov, \\ Godwin A. Ayoko (D) and Cheng Yan (D)*
}

\begin{abstract}
Lithium-sulfur ( $\mathrm{Li}-\mathrm{S})$ batteries are emerging as one of the promising candidates for next generation rechargeable batteries. However, dissolution of lithium polysulfides in the liquid electrolyte, low electrical conductivity of sulfur and large volume change during electrochemical cycling are the main technical challenges for practical applications. In this study, a systematic first-principles density functional theory calculation is adopted to understand the interactions between graphene and graphene with oxygen containing functional groups (hydroxyl, epoxy and carboxyl groups) and sulphur $\left(\mathrm{S}_{8}\right)$ and long chain lithium polysulfides $\left(\mathrm{Li}_{2} \mathrm{~S}_{8}\right.$ and $\left.\mathrm{Li}_{2} \mathrm{~S}_{4}\right)$. We find the adsorption is dominated by different mechanisms in sulphur and lithium polysulfides, i.e. van der Waals attraction and formation of coordinate covalent $\mathrm{Li}-\mathrm{O}$ bonds. The adsorption strength is dependent on the inter-layer distance and electron rich functional groups. Through these mechanisms, sulphur and lithium polysulfides can be successfully retained in porous graphene, leading to improved conductivity and charge transfer in the cathode of Li-S batteries.
\end{abstract}

\section{Introduction}

Recently, there has been an increasing demand for higher energy density in energy storage systems such as batteries and supercapacitors for portable devices and electric vehicles. With an energy capacity of $1673 \mathrm{~mA} \mathrm{~h} \mathrm{~g}^{-1}$ and a specific energy of $2600 \mathrm{~W} \mathrm{~h} \mathrm{~kg}^{-1}$, Li-S batteries are emerging as one of the promising candidates for next generation rechargeable batteries..$^{1-3}$ However, dissolution of lithium polysulfides in the liquid electrolyte, low electrical conductivity of sulfur and its final discharge products, and large volume change during electrochemical cycling are the main technical challenges. ${ }^{4,5}$ To solve these problems, recent efforts have been put into the design of nanostructured electrodes to improve their capacity and cycling performance. ${ }^{6,7}$ It has been confirmed that composite cathodes consisting of sulfur and nanostructured carbon materials such as meso-micro porous carbon, ${ }^{8}$ carbon spheres ${ }^{9,10}$ carbon nanotubes (CNTs), ${ }^{11}$ graphene, ${ }^{12-15}$ and graphene oxide, ${ }^{16,17}$ could mitigate the polysulfide shuttle via physical confinement of soluble polysulfides within the conductive carbonaceous structures. Among the various types of carbon materials, graphene has attracted much attention due to its good electrical conductivity and high surface area.

School of Chemistry, Physics and Mechanical Engineering, Faculty of Science and Engineering, Queensland University of Technology (QUT), Brisbane, QLD 4001, Australia.E-mail:c2.yan@qut.edu.au

$\dagger$ Electronic supplementary information (ESI) available. See DOI: 10.1039/c7ra11628d
Another alternative is reduced graphene oxide (rGO), a layered material with graphene domains and residual functional groups such as hydroxyl, epoxy and carboxyl groups. ${ }^{18,19}$ Consequently, attempts were made to design cathodes with porous graphene and $\mathrm{rGO}^{20-23}$ Chen's group ${ }^{24}$ employed a threedimensional rGO sponge to produce a sulfur nanogranular filmcoated composite cathode with a reversible capacity of $1080 \mathrm{~mA} \mathrm{~h} \mathrm{~g}^{-1}$ at $0.1 \mathrm{C}$ rate and $86.2 \%$ capacity retention after 500 cycles at $1.0 \mathrm{C}$ rate.

In General, microporous anchoring materials (pore size $<2 \mathrm{~nm}$ ) could successfully confine polysulfides to achieve good electro activity and long-term cyclability. ${ }^{25-27}$ However it was realized that anchoring materials with non-polar surfaces, alone cannot mitigate the polysulfide shuttle as they fail to make sufficient interaction with polar lithium polysulfides, and further surface modification is generally needed to chemically bind polysulfides onto the carbonaceous matrix. Recently, density functional theory (DFT) studies were carried out to investigate the discharge mechanisms in Li-S batteries ${ }^{28,29}$ and several investigators modelled the interactions between defective graphene, heteroatom doped graphene, and lithium polysulfides. ${ }^{30-32}$ However, the anchoring mechanisms of porous graphene functionalized with different oxygen groups during lithiation process have not been well understood. In addition, it is unclear if polar groups can facilitate the adsorption of nonpolar $\mathrm{S}_{8}$ as they interact with lithium polysulfides and if the functional groups can build up a barrier for electron transfer at the interface between $S_{8}$ and the substrate. 
In this paper, we report a systematic first-principle investigation on the anchoring effects of microporous epoxy-, hydroxyl- and carboxyl-functionalized graphene and elucidate the mechanisms responsive for interfacial interaction and electron transfer.

\section{Computational methods}

As shown in Fig. 1a, a basic structure with two parallel graphene layers is constructed to simulate porous graphene. The interlayer distance is changed in the range of 7.5-20 $\AA$ to understand how the pore size affects the adsorption of $\mathrm{S}$ compounds. The smallest interlayer distance (pore size) is set to $7.5 \AA$ to effectively accommodate the dimensions of cyclo- $S_{8}(\sim 0.7 \mathrm{~nm}){ }^{33,34}$ To model oxygen functionalized graphene (OFG) which consists of hydroxyl, epoxy and carboxyl functional groups, three distinct microporous structures are built using the basic model shown in Fig. 1a with a interlayer distance of $12.5 \AA$. For simplicity, they are referred as hG, eG and cG, respectively (Fig. 1b). Based on literature, ${ }^{35,36}$ hydroxyl and epoxy groups are introduced on the basal plane and a carboxyl group is introduced at the edge of the plane corresponding to oxygen atomic concentration of 1.4$2.6 \%$.

Molecule configurations of S compounds, adsorption energies of S compounds to microporous structures (graphene and OFG), charge transfer from $\mathrm{S}$ compounds at different lithiation stages, and density of states near Fermi energy region are examined using DFT calculations with DMol3 package p $^{37,38}$ of Materials Studio 2016. Electron-electron exchange correlations are described by generalized gradient approximations (GGA) of the Perdew-Burke-Ernzerhof (PBE) functional. ${ }^{39}$ To consider the van der Waals interaction, a semi-empirical dispersion potential of DFT-D2 method of Grimme is used. ${ }^{40}$ Energy, maximum force and maximum displacement are set to $2.0 \times$ $10^{-5}$ ha, $4 \times 10^{-3}$ ha $\mathrm{A}^{-1}$ and $5 \times 10^{-3} \mathrm{~A}$, respectively. Selfconsistent-field density is set to $1 \times 10^{-5} \mathrm{eV}$. Double numerical plus polarization (DNP) is selected as the basis set and effective core potentials (ECP) are employed to describe the core electrons. The corresponding $k$ point grid is generated by the Monkhrost-Pack technique ${ }^{\mathbf{4 1}}$ for the Brillouin zone sampling and simulations are carried out using a $5 \times 5 \times 1$ grid.

The binding energy ( $E_{\text {bind }}$ ) of each $\mathrm{Li}_{2} \mathrm{~S}_{x}$ molecules can be calculated as, $E_{\text {bind }}=\left[E_{\mathrm{Li}_{2} \mathrm{~S}_{x}}-\left(2 E_{\mathrm{Li}}+E_{\mathrm{S}_{x}}\right)\right] / 2$, where $E_{\mathrm{Li}_{2} \mathrm{~S}_{x}}, E_{\mathrm{Li}}$ and $E_{\mathrm{S}_{x}}$ are the energies of $\mathrm{Li}_{2} \mathrm{~S}_{x}, \mathrm{Li}$ atom and $\mathrm{S}_{x}$ molecule respectively. The adsorption energy ( $E_{\text {ads }}$ ) of each $S$ species to various graphene structures are calculated according to, $E_{\text {ads }}=E_{\text {Total }}-$ ( $\left.E_{\text {graphene }}+E_{\mathrm{S}}\right)$, where $E_{\text {Total }}, E_{\text {graphene }}$ and $E_{\mathrm{S}}$ are the total energies of the system, graphene structure and $\mathrm{S}$ containing cluster $\left(\mathrm{S}_{8}, \mathrm{Li}_{2} \mathrm{~S}_{8}\right.$ or $\left.\mathrm{Li}_{2} \mathrm{~S}_{4}\right)$, respectively. According to the equation, more negative adsorption energy indicates stronger interaction between graphene structure and S cluster. Initially, sulfur species were placed with different orientations inside the graphene structure. Eight different potential configurations of each $\mathrm{S}$ compound were considered and after relaxation, corresponding adsorption values were compared to obtain the most stable configuration (Fig. S1 $\dagger$ ). The energy difference between intact structure $\left(\mathrm{Li}_{2} \mathrm{~S}_{8} / \mathrm{Li}_{2} \mathrm{~S}_{4}\right)$ and decomposed structure ( $\mathrm{Li}+$ $\left.\mathrm{LiS}_{8} / \mathrm{Li}+\mathrm{LiS}_{4}\right)$ is calculated according to, $\Delta E=E\left(\mathrm{Li}_{2} \mathrm{~S}_{x}+\right.$ graphene $)-E\left(\mathrm{Li}+\mathrm{LiS}_{x}+\right.$ graphene $)$, where $x=4$ and 8 .

\section{Results and discussion}

\section{Effect of microporous graphene on adsorption of S species}

To find the structures with the minimum energy in $\mathrm{S}_{8}, \mathrm{Li}_{2} \mathrm{~S}_{8}$, $\mathrm{Li}_{2} \mathrm{~S}_{6}$ and $\mathrm{Li}_{2} \mathrm{~S}_{4}$, geometry optimization was carried out to obtain possible linear or closed atomic arrangements. ${ }^{30,42,43}$ Fig. 1c shows the stable ground state structures of $S_{8}$ and lithium polysulfides. Their bond lengths are consistent with previous investigations. ${ }^{30}$ The calculated bond length, charge of each atom via the Mulliken population analysis (MPA) and the binding energies of lithium polysulfides are shown in Table 1.

The most stable configuration of sulfur is the cycloocta-S which consists of covalently bonded 8 sulfur atoms in a crown formation with a calculated average S-S bond length of $2.092 \AA$. During the initial phase of the discharge process, $\mathrm{S}_{8}$ is reduced

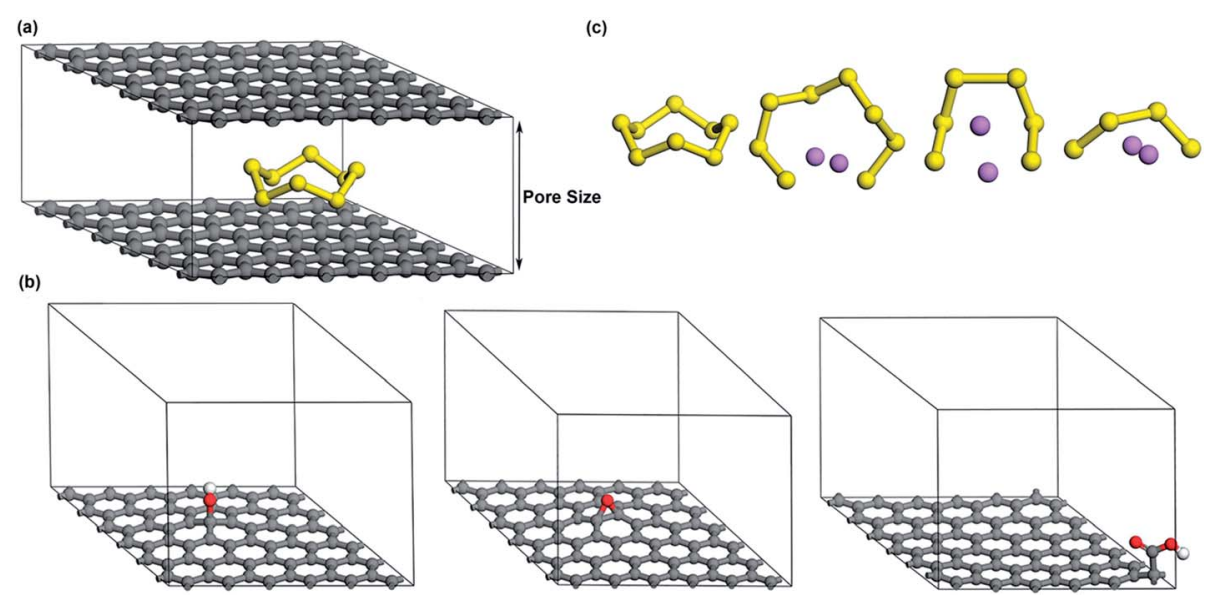

Fig. 1 (a) Microporous graphene (b) microporous structures of $h G$, eG and cG respectively (c) geometrically optimized minimum energy structures of $\mathrm{S}_{8}, \mathrm{Li}_{2} \mathrm{~S}_{8}, \mathrm{Li}_{2} \mathrm{~S}_{6}$ and $\mathrm{Li}_{2} \mathrm{~S}_{4}$ respectively (carbon, sulfur, oxygen, hydrogen and lithium atoms are represented by grey, yellow, red, white and purple, respectively). 
Table 1 Average bond distances, average charges and binding energies

\begin{tabular}{llll}
\hline & $\mathrm{Li}_{2} \mathrm{~S}_{8}$ & $\mathrm{Li}_{2} \mathrm{~S}_{6}$ & $\mathrm{Li}_{2} \mathrm{~S}_{4}$ \\
\hline $\mathrm{Li}-\mathrm{S}(\AA)$ & 2.450 & 2.429 & 2.405 \\
$\mathrm{~S}-\mathrm{S}(\AA)$ & 2.103 & 2.138 & 2.116 \\
Charge on Li & 0.44 & 0.44 & 0.46 \\
Binding energy $(\mathrm{eV})$ & -3.39 & -4.03 & -3.50
\end{tabular}

to $\mathrm{S}_{8}{ }^{2-}, \mathrm{S}_{6}{ }^{2-}, \mathrm{S}_{4}{ }^{2-}$ forming high-order lithium polysulfides and further gets reduced to $\mathrm{S}_{2}{ }^{2-}$ and $\mathrm{S}^{2-}$ forming low-order lithium polysulfides at the latter stages. ${ }^{2}$ According to our calculations, as $x$ in $\mathrm{Li}_{2} \mathrm{~S}_{x}$ decreases from 8 to 4 , the average $\mathrm{Li}-\mathrm{S}$ distance decreases from $2.450 \AA$ to $2.405 \AA$, implying that the interaction between $\mathrm{Li}$ and $\mathrm{S}$ atom strengthens as the $x$ decreases. The average S-S bond lengths of $\mathrm{Li}_{2} \mathrm{~S}_{8}, \mathrm{Li}_{2} \mathrm{~S}_{6}$ and $\mathrm{Li}_{2} \mathrm{~S}_{4}$ are 2.103, 2.138 and $2.118 \AA$, respectively. Our results revealed that $\mathrm{S}-\mathrm{S}$ bond lengths of polysulfides are larger than that of $\mathrm{S}_{8}$ molecule which is $2.092 \AA$ due to the less covalent nature of the $\mathrm{S}$ cluster within the lithium polysulfide. Due to the weak interaction between $\mathrm{Li}$ and $\mathrm{S}$, high order polysulfides tend to ionize easily into Li and polysulfide ions in the electrolyte than low-order $\mathrm{Li}_{2} \mathrm{~S}_{2}$ and $\mathrm{Li}_{2} \mathrm{~S}$. MPA is a qualitative method to understand how the charge is distributed among each atom. According to

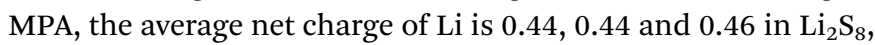
$\mathrm{Li}_{2} \mathrm{~S}_{6}$ and $\mathrm{Li}_{2} \mathrm{~S}_{4}$ respectively. Therefore, the interaction between $\mathrm{Li}$ and $\mathrm{S}$ in $\mathrm{Li}_{2} \mathrm{~S}_{8}$ is the weakest due to the largest $\mathrm{Li}-\mathrm{S}$ distance and the lowest average net charge of Li. Moreover, $\mathrm{Li}_{2} \mathrm{~S}_{8}$ exhibits the lowest binding energy of $-3.39 \mathrm{eV}$, suggesting that it is the most soluble compound among these lithium polysulfides during a charge/discharge cycle, consistent with previous experiments. ${ }^{44}$ As an intermediate which presents in both high and low voltage plateau regimes of charge/discharge cycle, $\mathrm{Li}_{2} \mathrm{~S}_{4}$ plays a vital role in the Li-S redox reaction. Elemental S is the starting point of the multi-electron-transfer cathode reaction, and hence the retention of $\mathrm{S}_{8}$ is important for the long lasting performance of a $\mathrm{Li}-\mathrm{S}$ cell. For these reasons, the interaction between $\mathrm{S}_{8}, \mathrm{Li}_{2} \mathrm{~S}_{8}$ and $\mathrm{Li}_{2} \mathrm{~S}_{4}$ and the porous graphene deserves further investigation.

As shown in Fig. 2a (Table S1 $\dagger$ ), the variation of energy profiles indicates that the adsorption energy $\left(E_{\text {ads }}\right)$ increases as the pore size decreases. The adsorption energy of S compounds to a typical anchoring material of the cathode depends on (1) the chemical interaction between the lithium polysulfide and the anchoring material in which a covalent bond can be formed between the $\mathrm{Li}$ atom in $\mathrm{Li}_{2} \mathrm{~S}_{x}$ and the functional group of the anchoring material and/or between the $\mathrm{S}$ atom of $\mathrm{Li}_{2} \mathrm{~S}_{x}$ and the functional group of the anchoring material and (2) the physical van der Waals attraction. Unlithiated $\mathrm{S}_{8}$ is a non-polar molecule, and does not form any chemical interaction with graphene domains. Consequently, the interfacial interaction is mainly governed by the physical interaction. However, as the lithiation begins, apart from the physical interaction, a chemical interaction between $\mathrm{Li}$ atoms and the anchoring material is formed. In graphene, regardless of different lithiation stages the physical interaction overpowers the chemical interaction and there

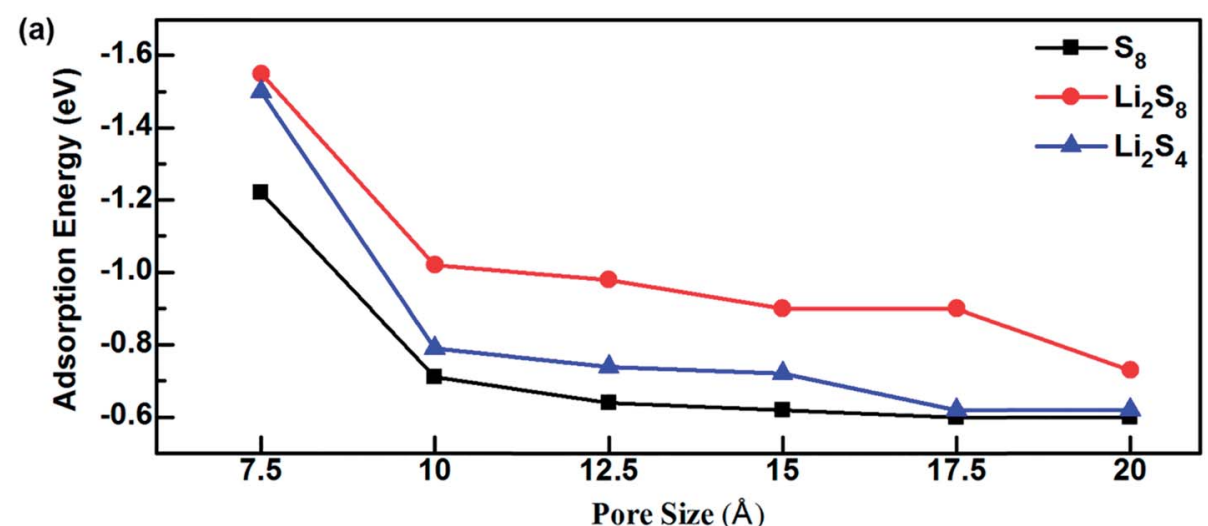

(b)

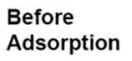
Adsorption
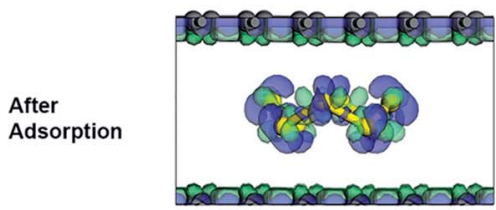

(c)
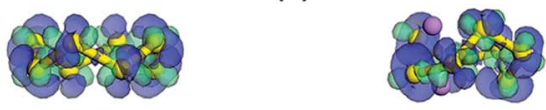

(d)

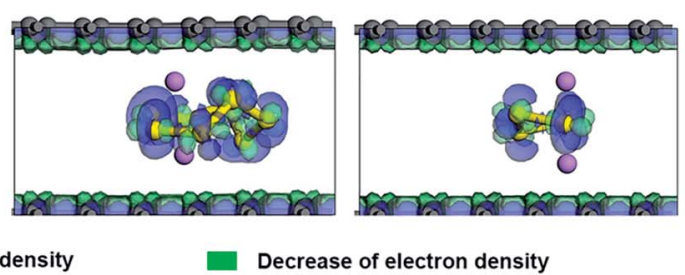

Fig. 2 (a) Adsorption energies of $\mathrm{S}_{8}, \mathrm{Li}_{2} \mathrm{~S}_{8}$ and $\mathrm{Li}_{2} \mathrm{~S}_{4}$ to microporous graphene. Deformation charge density and adsorption site of (b) $\mathrm{S}_{8}$, (c) $\mathrm{Li}_{2} \mathrm{~S}_{8}$, and (d) $\mathrm{Li}_{2} \mathrm{~S}_{4}$. 
is a significant increase of adsorption strength as the pore size decreases from $10 \AA$ to $7.5 \AA$. When the pore size is $7.5 \AA$, the adsorption energies are $-1.55 \mathrm{eV},-1.50 \mathrm{eV}$ and $-1.22 \mathrm{eV}$ for $\mathrm{Li}_{2} \mathrm{~S}_{8}, \mathrm{Li}_{2} \mathrm{~S}_{4}$ and $\mathrm{S}_{8}$, respectively.

The effect of van der Waals interaction on the confinement of $\mathrm{S}$ species inside microporous graphene can be visualized from the deformation charge density analysis (Fig. 2b-d), in which the increase and decrease of total electron density are denoted in blue and green respectively. The electron density difference is expressed as, $\Delta \rho=\rho_{\text {Total }}-\left(\rho_{\text {graphene }}+\rho_{\mathrm{S}}\right)$, where $\rho_{\text {Total }}, \rho_{\text {graphene }}$ and $\rho_{\mathrm{S}}$ are the electron densities of the system, graphene structure and the S-containing cluster respectively. It can be seen that charge is transferred inside the $\mathrm{S}$ species and inside the graphene surfaces, but no apparent charge transfer occurs between them, suggesting no strong chemical interaction. However, by thoroughly comparing the deformation charge density of $\mathrm{Li}_{2} \mathrm{~S}_{8}$ and $\mathrm{Li}_{2} \mathrm{~S}_{4}$ with their adsorption sites (Fig. $2 \mathrm{c}-\mathrm{d}$ ), it can be seen that $\mathrm{Li}$ atoms in $\mathrm{S}$ complexes have slightly moved away from $\mathrm{S}$ and towards the graphene surface, indicating a slight chemical attraction between $\mathrm{Li}$ and $\mathrm{C}$ atoms which explains why $\mathrm{Li}_{2} \mathrm{~S}_{8}$ and $\mathrm{Li}_{2} \mathrm{~S}_{4}$ exhibit higher adsorption energy values than $S_{8}$. Due to the fact that unlithiated $S_{8}$ is confined inside a narrow pore which induces a strong physical interaction from both sides of the graphene surfaces, the adsorption value of $-1.22 \mathrm{eV}$ exhibited by microporous graphene with a pore size of $7.5 \AA$ is much higher than values recorded for anchoring materials like $\mathrm{V}_{2} \mathrm{O}_{5}, \mathrm{MoS}_{2}$ and phosphorene. ${ }^{45,46}$

\section{Influence of different functional groups of OFG towards adsorption of $\mathbf{S}$ species}

The interactions between $\mathrm{S}_{8}, \mathrm{Li}_{2} \mathrm{~S}_{4}$ and $\mathrm{Li}_{2} \mathrm{~S}_{8}$ molecules with functional groups $\mathrm{hG}$, eG and $\mathrm{cG}$, were simulated and the adsorption energies are summarized in Fig. 3 (Table $\mathrm{S} 2 \dagger$ ). Fig. 4a shows the final optimized structures of $S_{8}$ on pure graphene, hG, eG and cG, respectively. Note that in all the cases, the highest adsorption energy was given when $\mathrm{S}_{8}$ adsorbed from the opposite direction of the functional group and parallel to the graphene surface. According to our calculations, it was found that when compared with graphene, hG, eG and cG exhibit almost similar adsorption energy to $S_{8}$, illustrating that oxygen functional groups do not have a major influence on the adsorption of $\mathrm{S}_{8}$, as the adsorption strength is dominated by van der Waals attraction.

To further investigate the interaction between $\mathrm{S}_{8}$ and porous graphene, MPA is applied to determine how much charge transfer has occurred between them. Interestingly, 0.09 electrons have been transferred from $S_{8}$ to the graphene surface but there is only a minor contribution to charge transfer between $S_{8}$ and OFG where only $0.04,0.03$ and 0.06 electrons have been withdrawn from hG, eG and cG respectively (Fig. 4b). Fig. 4c shows the density of states (DOS) near Fermi energy $\left(E_{\mathrm{f}}\right)$ for the adsorption system of $\mathrm{S}_{8}$ in graphene and OFG, in which the $E_{\mathrm{f}}$ is set to $0 \mathrm{eV}$ and represented by a vertical dotted line. For $\mathrm{S}_{8}$ adsorbed graphene system, the DOS value at $E_{\mathrm{f}}$ is found to be 4.33 electrons per $\mathrm{eV}$ and it reduces to 4.00 and 3.92 electrons

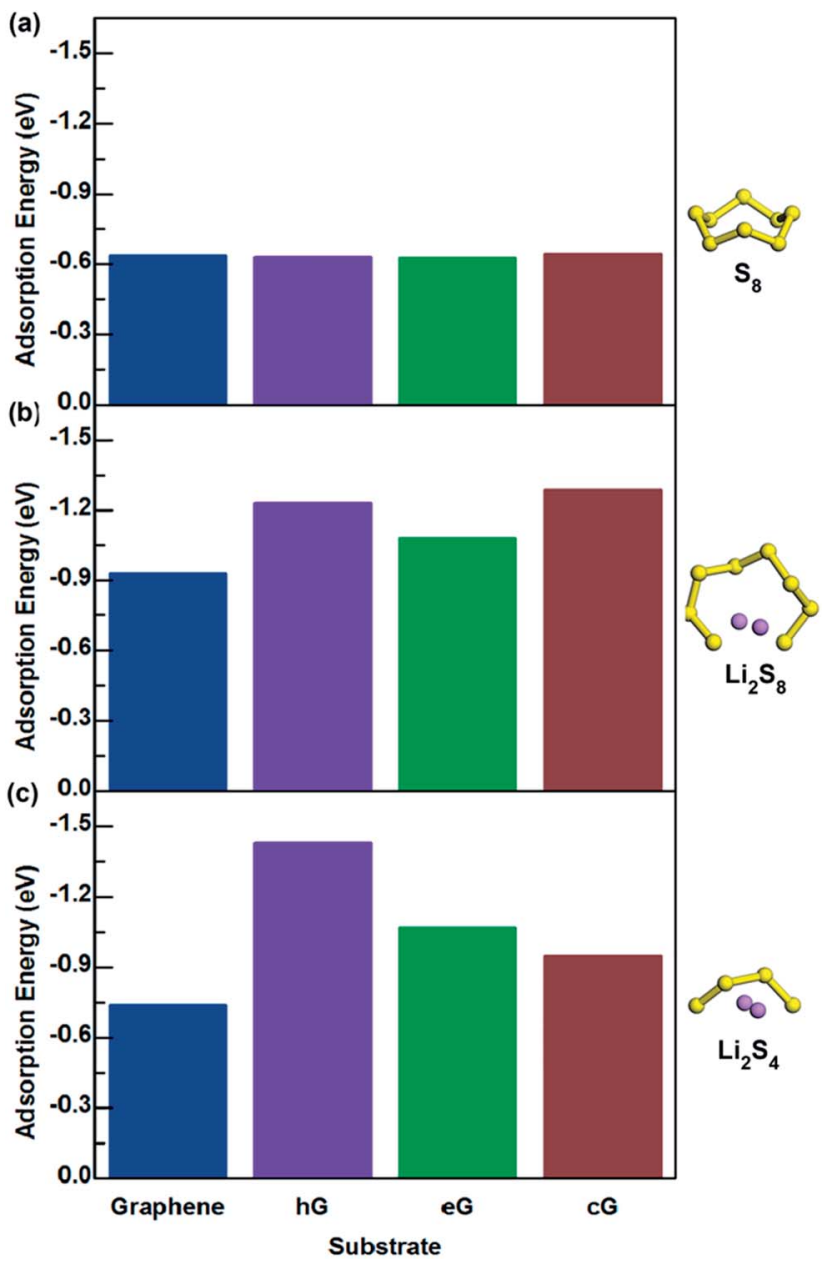

Fig. 3 Adsorption values of (a) $\mathrm{S}_{8}$, (b) $\mathrm{Li}_{2} \mathrm{~S}_{8}$, and (c) $\mathrm{Li}_{2} \mathrm{~S}_{4}$ interacting with graphene, hG, eG and cG.

per eV when $\mathrm{S}_{8}$ gets adsorbed to hG and $\mathrm{cG}$, respectively (Table $\mathrm{S} 3 \dagger)$. Furthermore DOS value at $E_{\mathrm{f}}$ drops to 3.15 electrons per eV when eG adsorbs $\mathrm{S}_{8}$, suggesting that electron transfer at the interface is slightly hindered due to the oxygen functional groups on the graphene surface which then leads to building up of ohmic resistance at the $\mathrm{S}_{8} / \mathrm{OFG}$ interface. This phenomenon is also reported by Shiqi et al. where they discovered that $S_{8}$ is decoupled from the graphitic surface due to the presence of Triton X-100, a non-ionic surfactant with a polyethylene oxide chain. ${ }^{47}$

As for the adsorption of $\mathrm{Li}_{2} \mathrm{~S}_{8}$ molecule, cG exhibits the highest adsorption energy of $-1.29 \mathrm{eV}$ and all the oxygen functional groups show significantly higher adsorption energies compared to pristine graphene (Fig. 3b). When interacting with $\mathrm{Li}_{2} \mathrm{~S}_{4}$, hG exhibits the highest adsorption energy of $-1.43 \mathrm{eV}$ and it is almost over 2 times higher than pristine graphene (Fig. 3c). Fig. 5b-i shows the optimized structures of $\mathrm{Li}_{2} \mathrm{~S}_{8}$ and $\mathrm{Li}_{2} \mathrm{~S}_{4}$ on hG, eG and cG, respectively. To further get an understanding of the magnitude of the $\mathrm{Li}-\mathrm{O}$ interaction, we optimized the geometry of a $\mathrm{Li}_{2} \mathrm{O}$ crystal (Fig. $\mathrm{S} 2 \dagger$ ) so that it could be used as a benchmark to compare the bonding nature of $\mathrm{Li}$ and $\mathrm{O}$ in the optimized models. ${ }^{48}$ The calculated Li-O distances of 
(a)

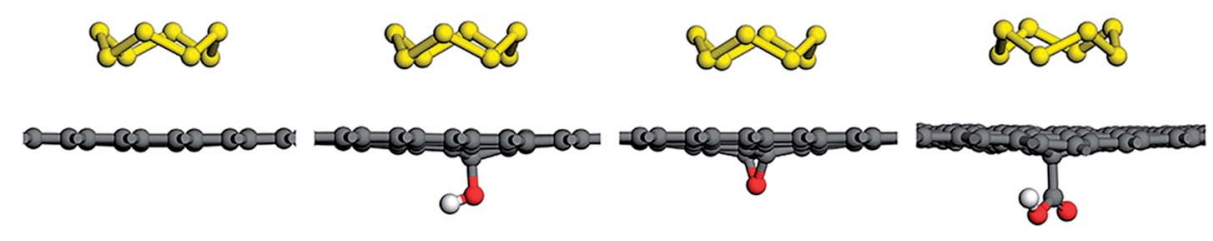

(b)

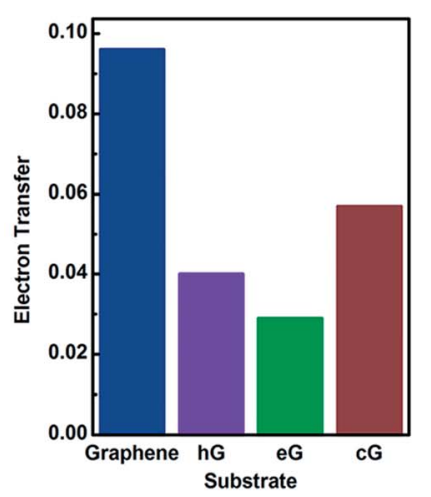

(c)

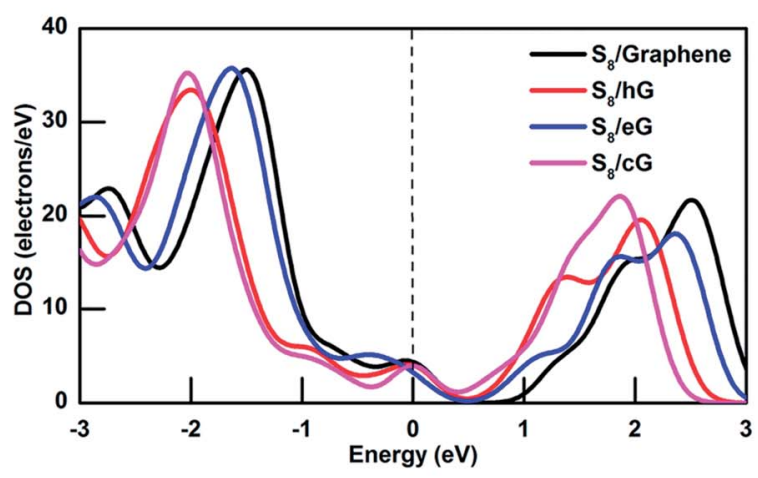

Fig. 4 (a) $\mathrm{S}_{8}$ adsorption on graphene, $h G, e G$ and cG. Oxygen and hydrogen atoms are represented by red and white respectively. (b) Electron transfer from $\mathrm{S}_{8}$ to different substrates, and (c) density of states near Fermi energy region for $\mathrm{S}_{8}$ on graphene, $h G, e G$ and $c G$.

(a)

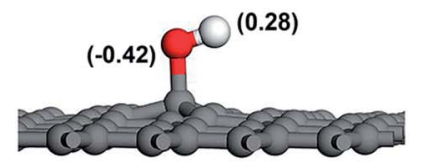

(b)

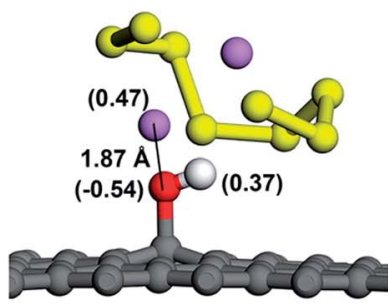

(c)

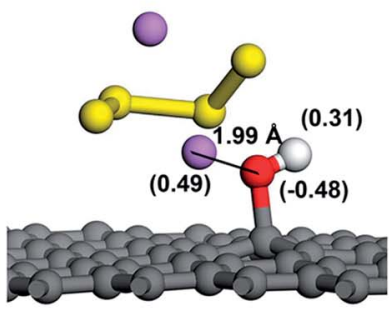

(d)

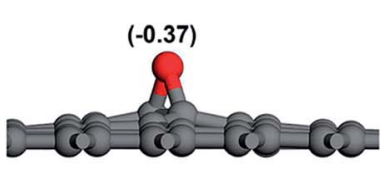

(e)
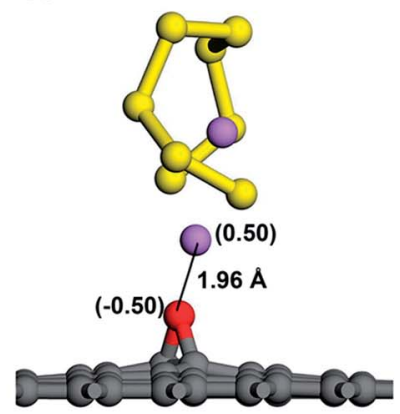

(f)

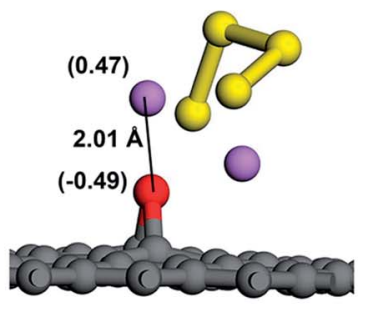

(g)

$(-0.37)$

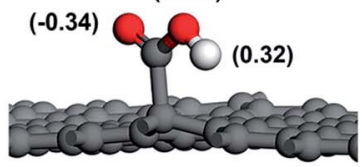

(h)

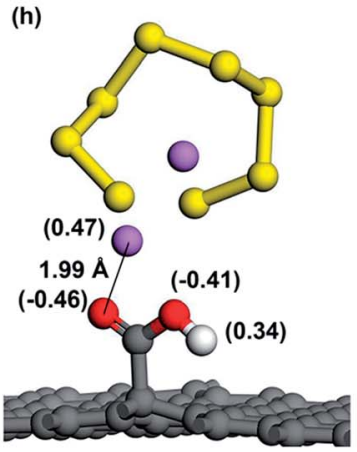

(i)

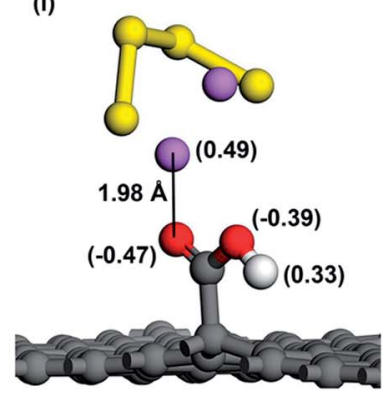

Fig. 5 Mulliken charge distribution before and after adsorption of $\mathrm{Li}_{2} \mathrm{~S}_{8}$ and $\mathrm{Li}_{2} \mathrm{~S}_{4}$ of $(\mathrm{a}-\mathrm{c}) \mathrm{hG}$, (d-f) eG and (g-i) cG respectively. Note that charge of each atom is shown in parentheses. The shortest distance between $\mathrm{Li}$ atom and $\mathrm{O}$ atom is also shown in angstroms ( $(\AA)$ ). 
adsorption geometries are almost similar with the strong ionic bond length of $\mathrm{Li}_{2} \mathrm{O}$ molecule implying the existence of a strong attraction between $\mathrm{Li}$ and $\mathrm{O}$ in all the cases. hG had the shortest Li-O distance of $1.87 \AA$ when it interacted with $\mathrm{Li}_{2} \mathrm{~S}_{8}$ while the longest $\mathrm{Li}-\mathrm{O}$ distance of $2.01 \AA$ was given by eG when it interacted with $\mathrm{Li}_{2} \mathrm{~S}_{4}$ (Table $\mathrm{S} 4 \dagger$ ).

As $\mathrm{O}$ atom has a high electronegativity of 3.44 and $\mathrm{Li}$ atom has a low electronegativity of 0.98 , the strong attraction between $\mathrm{Li}$ and $\mathrm{O}$ can be explained by the Lewis-acid base theory. Since epoxy, hydroxyl and carboxyl groups all consist of $\mathrm{O}$ atoms with lone electron pairs in their outer p orbitals; they act as electron pair donors (Lewis bases). These Lewis-base sites are attracted by the terminal $\mathrm{Li}$ atoms of $\mathrm{Li}_{2} \mathrm{~S}_{8}$ and $\mathrm{Li}_{2} \mathrm{~S}_{4}$ which act as strong Lewis acids according to the Lewis-acid base theory. Fig. 6 shows the deformation charge density corresponding to $\mathrm{Li}_{2} \mathrm{~S}_{8}$ and $\mathrm{Li}_{2} \mathrm{~S}_{4}$ adsorption sites. A significantly high electron density is visible around the lone pairs of the $\mathrm{O}$ atom strengthening the fact that extra pairs of electrons act as electron rich donor to interact with strong Lewis acid of $\mathrm{Li}$ ion to form a coordinate covalent bond. The strong attraction between negatively charged oxygen atom and the positively charged $\mathrm{Li}$ atom can be further illustrated in Fig. 5 where the Mulliken charge distribution of hG, eG and cG before and after adsorption of $\mathrm{Li}_{2} \mathrm{~S}_{8}$ and $\mathrm{Li}_{2} \mathrm{~S}_{4}$ are presented. Because of the polarization of the $\mathrm{O}$ atom by the terminal $\mathrm{Li}$ atom of $\mathrm{Li}_{2} \mathrm{~S}_{8}, 0.12,0.13$ and 0.12 electrons are withdrawn by the closest $\mathrm{O}$ atom of each eG, hG and cG respectively. The same phenomenon occurs when those substrates interact with $\mathrm{Li}_{2} \mathrm{~S}_{4}$ where $0.06,0.12$ and 0.13 electrons have been transferred to the $\mathrm{O}$ atom. Therefore functionalized carbon materials consisting of highly electronegative atoms with lone electron pairs, are good candidates for the immobilization of high order lithium polysulfides due to the moderate adsorption ability. Recently, a composite cathode made out of chlorine-reinforced carbon nanofibers reported to have enhanced cycling performance, validating our theoretical predictions. ${ }^{49}$

Though there is a relatively strong interaction between lithium polysulfides and the OFG, the polysulfide molecule itself remains intact without being dissociated into short chain lithium polysulfides upon adsorption. We observed that after adsorption, the internal $\mathrm{Li}-\mathrm{S}$ bond length increased slightly from $2.45 \AA$ to $2.48 \AA$ in $\mathrm{Li}_{2} \mathrm{~S}_{8}$ and $2.41 \AA$ to $2.46 \AA$ in $\mathrm{Li}_{2} \mathrm{~S}_{4}$ (Table $\mathrm{S} 4 \dagger)$. Various layered metal oxides and sulfides were reported to induce a strong chemical bond with lithium polysulfides in terms of $\mathrm{Li}-\mathrm{O}, \mathrm{Li}-\mathrm{S}$ or $\mathrm{M}-\mathrm{S}$ bonds ( $\mathrm{M}$ represents metal oxides/ sulfides). ${ }^{\mathbf{5 0 , 5 1}}$ However, recently it was discovered that strong interaction with polysulfides could interfere on $\mathrm{S}$ reduction reactions and sometimes weaken the $\mathrm{Li}-\mathrm{S}$ bond causing the dissociation of the $\mathrm{Li}_{2} \mathrm{~S}_{x}$ molecule. Such separation between $\mathrm{Li}$ and $\mathrm{S}$ atom eventually could lead up to the formation of $\mathrm{Li}^{+}$and $\mathrm{S}_{x}{ }^{2-}$ ions and as a result sulfur could be dissolved in the electrolyte adversely affecting the performance of the $\mathrm{Li}-\mathrm{S}$ cell. ${ }^{\mathbf{4 9 , 5 0 , 5 2}}$ Furthermore, Yu et al. revealed the importance of employing a cathode material with a moderate binding capability which allows a small amount of polysulfide dissolution in order to improve the stability of the solid electrolyte interface (SEI) on the lithium anode. ${ }^{49}$

To further investigate the possibility of the decomposition of $\mathrm{Li}_{2} \mathrm{~S}_{x}$ by the weakening of the $\mathrm{Li}-\mathrm{S}$ bond due to the attraction of OFG, we considered the adsorption of the decomposed $\mathrm{LiS}_{8}$ and (a)

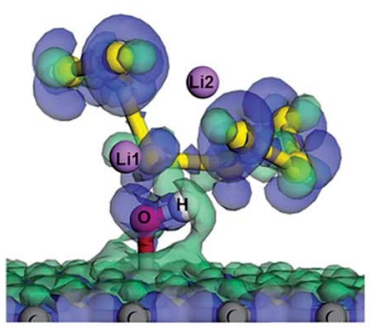

(b)

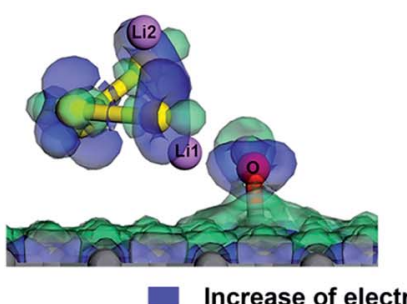

(c)

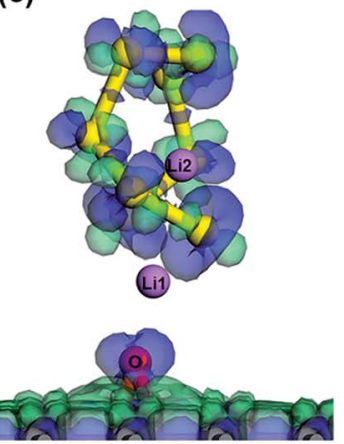

(d)

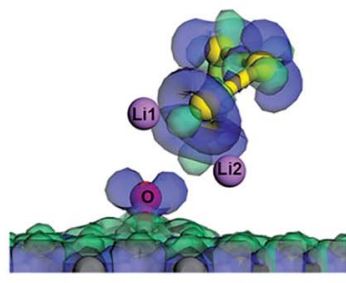

(e)

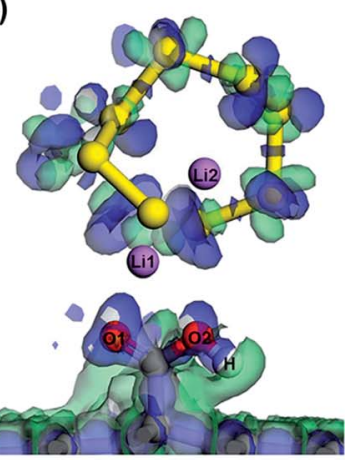

(f)

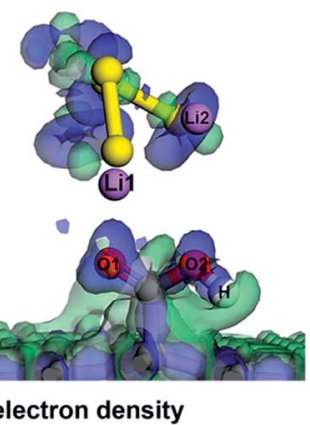

Fig. 6 Deformation charge density at $\mathrm{Li}_{2} \mathrm{~S}_{8}$ and $\mathrm{Li}_{2} \mathrm{~S}_{4}$ adsorption sites of (a and b) hG, (c and d) eG and (e and f) cG. (The increase/decrease of electron density is denoted by blue/green, respectively.) 
$\mathrm{LiS}_{4}$ structures along with an isolated $\mathrm{Li}$ atom and calculated the energy difference $(\Delta E)$ between the decomposed structure and the intact structure $\left(\mathrm{Li}_{2} \mathrm{~S}_{8}\right.$ and $\left.\mathrm{Li}_{2} \mathrm{~S}_{4}\right)$. A negative value for $\Delta E$ indicates that the intact structure has lower energy than the decomposed structure and the intact structure is energetically stable. Fig. 7 shows the adsorbed $\mathrm{Li}+\mathrm{LiS}_{8}$ and $\mathrm{Li}+\mathrm{LiS}_{4}$ clusters on graphene, hG, eG and cG. According to $\Delta E$ values in all the cases, the intact structure proved to be energetically preferable over the decomposed structure. Therefore microporous graphene decorated with oxygen functional groups could be considered as an effective choice as a cathode material for $\mathrm{Li}-\mathrm{S}$ batteries which strikes a balance between adsorption strength and intactness of high order lithium polysulfides.

To gain further insights into the bond interaction between OFG and $\mathrm{Li}_{2} \mathrm{~S}_{8} / \mathrm{Li}_{2} \mathrm{~S}_{4}$, we analysed the atomic partial density of states (PDOS) near $E_{\mathrm{f}}$ for the adsorption systems (Fig. S3 $\uparrow$ ). The PDOS for Li-1 and O-1 atoms (indicated by black and blue colour) are seen to overlap at the upper part of the valence band (just below the Fermi level) suggesting that there is a hybridization between Li-2s and $\mathrm{O}-2 \mathrm{p}$ orbitals. This further ascertains the existence of a $\mathrm{Li}-\mathrm{O}$ covalent bond which enhances the interaction between the substrate and the polysulfides and thereby mitigates the polysulfide shuttle effectively.

The DOS near Fermi energy for hG, eG and $\mathrm{cG}$ before and after the anchoring of $\mathrm{S}_{8}, \mathrm{Li}_{2} \mathrm{~S}_{8}$ and $\mathrm{Li}_{2} \mathrm{~S}_{4}$ are presented in Fig. $\mathrm{S} 4, \dagger$ in which hG, eG and cG exhibit metallic nature with $3.98,3.16$ and 3.92 electrons per eV DOS values at $E_{\mathrm{f}}$ respectively at oxygen atomic concentrations of $1.4-2.6 \%$. The good electrical conductivity of reduced graphene oxide has also been demonstrated by previous computational studies ${ }^{53,54}$ and, furthermore Stankovich et al. ${ }^{55}$ produced rGO nanosheets with a significantly high conductivity value of $\left(\sim 2 \times 10^{2} \mathrm{~S} \mathrm{~m}^{-1}\right)$ which closely approaches that of pristine graphene, even at an atomic $\mathrm{C} / \mathrm{O}$ ratio of $\sim 10$.

The strong affinity towards $\mathrm{Li}_{2} \mathrm{~S}_{8} / \mathrm{Li}_{2} \mathrm{~S}_{4}$ by the OFG is clearly observed from the DOS values of $\mathrm{hG}$, eG and cG after anchoring of $\mathrm{Li}_{2} \mathrm{~S}_{8}$ and $\mathrm{Li}_{2} \mathrm{~S}_{4}$. When $\mathrm{Li}_{2} \mathrm{~S}_{8}$ and $\mathrm{Li}_{2} \mathrm{~S}_{4}$ are adsorbed, due to the newly formed $\mathrm{Li}-\mathrm{O}$ covalent bond between $\mathrm{Li}$ atom of $\mathrm{Li}_{2} \mathrm{~S}_{8} /$ $\mathrm{Li}_{2} \mathrm{~S}_{4}$ and $\mathrm{O}$ atom of the functional groups, more electrons are transferred to the adsorption sites and the DOS curve exhibits a higher value at $E_{\mathrm{f}}$ when compared with the $\mathrm{S}_{8}$ adsorbed system. Therefore polar groups maintain a strong interaction with the lithium polysulfides in the discharge/charge cycle of the Li-S battery and in the meantime improve the electrical conductivity of the cathode by facilitating the charge transfer at the interface.

In a typical Li-S battery, the organic electrolyte consists of bis(trifluoromethane)sulfonimide lithium salt (LiTFSI) dissolved in a mixture of 1,3-dioxolane (DOL) and 1,2-dimethoxyethane (DME). To investigate the influence of the electrolyte towards the discharge/charge cycle of the battery, we calculated the adsorption energy values of DOL and DME towards $\mathrm{S}_{8}, \mathrm{Li}_{2} \mathrm{~S}_{8}$ and $\mathrm{Li}_{2} \mathrm{~S}_{4}$ (Fig. 8a) and compared them with the values we gained for graphene and OFG. For ease of comparison, we used the average adsorption values of $\mathrm{hG}$, eG, and $\mathrm{cG}$ as the adsorption value for OFG in each lithiation stage. According to Fig. 8b, adsorption energies of graphene and OFG towards $\mathrm{S}_{8}$ are almost similar and they are significantly higher than DOL and DME, suggesting that van der Waals interaction is more than sufficient to retain $S_{8}$ in the cathode. However $\mathrm{Li}_{2} \mathrm{~S}_{8}$ and $\mathrm{Li}_{2} \mathrm{~S}_{4}$ are barely adsorbed by graphene and there's a high possibility of these long chain polysulfides being dissolved in the liquid electrolyte. When compared with DOL and DME, adsorption energy of OFG towards $\mathrm{Li}_{2} \mathrm{~S}_{8} / \mathrm{Li}_{2} \mathrm{~S}_{4}$ is significantly high proving the fact that functional groups could confine lithium polysulfides inside the cathode and mitigate the shuttle effect.

Based on the insights gained from our theoretical study, we can conclude that adsorption strength and electron transfer at the interface are two critical aspects which have to be considered when selecting an anchoring material for the cathode in $\mathrm{Li}-\mathrm{S}$ batteries. At the initial stage of the discharge cycle, confinement of $S_{8}$ is controlled by the van der Waals interaction, and it is highly unlikely for $S_{8}$ being dissolved in the electrolyte due to the poor attraction of the solvents towards $\mathrm{S}_{8}$ molecules. A microporous substrate is more suitable to confine $\mathrm{S}_{8}$ as it can be confined inside a narrow pore which induces a strong physical interaction. Surface functionalization of graphene has no additional influence on attracting $S_{8}$ to its surface since almost no chemical interaction can be formed between functional groups and non-polar $\mathrm{S}_{8}$. Furthermore functional groups adversely affect the electron transfer and increase the ohmic resistance at the substrate $/ S_{8}$ interface. However functional groups have a major impact on mitigating the shuttle effect and improving the capacity retention of $\mathrm{Li}-\mathrm{S}$ cell by

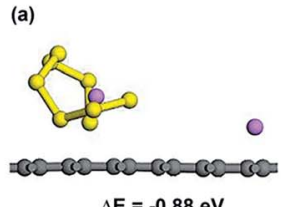

(b)

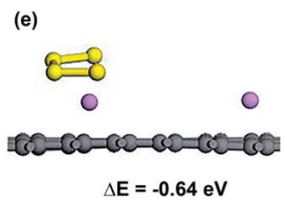

(f)

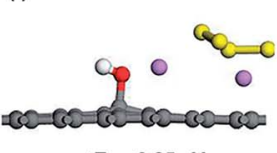

(c)

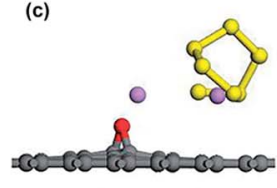

$\Delta E=-0.76 \mathrm{eV}$

(g)

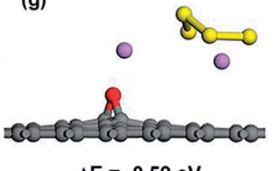

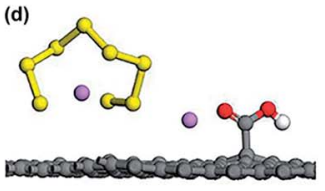

$\Delta \mathrm{E}=-\mathbf{0 . 8 9} \mathrm{eV}$

(h)

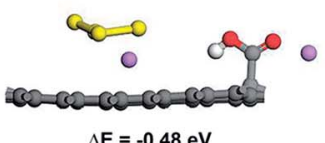

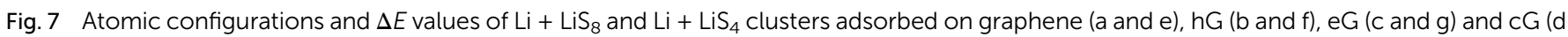
and $h$ ) respectively. 
(a)
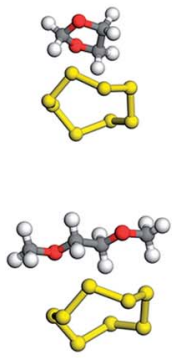
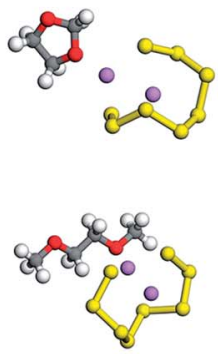

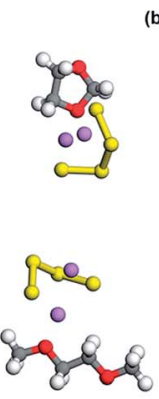

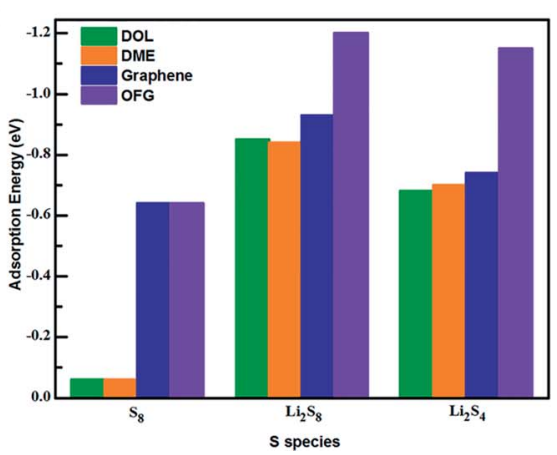

Fig. 8 (a) Interaction of $\mathrm{S}_{8}, \mathrm{Li}_{2} \mathrm{~S}_{8}$ and $\mathrm{Li}_{2} \mathrm{~S}_{4}$ with DOL and DME. (b) Adsorption energies of $\mathrm{S}_{8}$, $\mathrm{Li}_{2} \mathrm{~S}_{8}$ and $\mathrm{Li}_{2} \mathrm{~S}_{4}$ interacting with DOL, DME, graphene and OFG.

ensuring a strong interaction with $\mathrm{Li}_{2} \mathrm{~S}_{8} / \mathrm{Li}_{2} \mathrm{~S}_{4}$. On the other hand, the strong interaction with lithium polysulfides facilitates the interfacial electron transfer through the $\mathrm{Li}-\mathrm{O}$ bond which can be explained by the Lewis-acid base theory.

\section{Conclusions}

In this work, we have investigated the interactions between graphene with oxygen containing functional groups (hydroxyl, epoxy and carboxyl groups) and sulphur $\left(\mathrm{S}_{8}\right)$ and long chain lithium polysulfides $\left(\mathrm{Li}_{2} \mathrm{~S}_{8}\right.$ and $\left.\mathrm{Li}_{2} \mathrm{~S}_{4}\right)$, via density functional theory computations. During the initial unlithiated stage, we find the interaction between sulphur and pristine graphene and OFG are dominated by van der Waals attraction. Although graphene and OFG exhibits almost the same adsorption capability towards nonpolar $\mathrm{S}_{8}$, the functional groups develop a slight barrier for electron transfer at the interface and increase the ohmic resistance. The highest adsorption energy is observed when the distance between two graphene layers approaches to about 7.5 A. During the lithiation stage, surface functionalization of graphene significantly enhances the interaction with $\mathrm{Li}_{2} \mathrm{~S}_{8} / \mathrm{Li}_{2} \mathrm{~S}_{4}$ by forming a coordinate covalent $\mathrm{Li}-\mathrm{O}$ bond. Due to the covalent nature of the $\mathrm{Li}-\mathrm{O}$ bond, polysulfides are well retained inside the cathode and it also improves the conductivity of the electrode upon the deposition of $\mathrm{Li}_{2} \mathrm{~S}_{8}$ and $\mathrm{Li}_{2} \mathrm{~S}_{4}$ by facilitating the interfacial charge transfer. Furthermore, our work explains the reason why porous graphene with oxygen functional groups is more effective as a cathode material compared to materials with too strong interactions which could cause destruction effects on the adsorbed lithium polysulfides. Due to the moderate binding affinity, OFG strikes a balance between adsorption strength and intactness of high order lithium polysulfides. Therefore based on our simulations, we suggest that microporous graphene decorated with hydroxyl, epoxy and carboxyl functional groups can successfully anchor sulfur and lithium polysulfides via moderate interactions, leading to improved conductivity and charge transfer in the cathode of Li-S batteries.

\section{Conflicts of interest}

The authors declare no conflict of interest.

\section{Acknowledgements}

The authors wish to acknowledge the High Performance Computing and Research Support (HPC) group in Queensland University of Technology for access to its computation resources. KW would like to acknowledge Queensland University of Technology for financial support via APA and IPRS scholarships. The financial support from an ARC Discovery Project (DP150101717) is appreciated.

\section{References}

1 A. Manthiram, S.-H. Chung and C. Zu, Adv. Mater., 2015, 27, 1980-2006.

2 Z. Lin and C. Liang, J. Mater. Chem. A, 2015, 3, 936-958.

3 A. Manthiram, Y. Fu, S.-H. Chung, C. Zu and Y.-S. Su, Chem. Rev., 2014, 114, 11751-11787.

4 D.-W. Wang, Q. Zeng, G. Zhou, L. Yin, F. Li, H.-M. Cheng, I. R. Gentle and G. Q. M. Lu, J. Mater. Chem. A, 2013, 1, 9382-9394.

5 H. Kim, H.-D. Lim, J. Kim and K. Kang, J. Mater. Chem. A, 2014, 2, 33-47.

6 M. A. Pope and I. A. Aksay, Adv. Energy Mater., 2015, 5, 1500124.

7 L. Ma, K. E. Hendrickson, S. Wei and L. A. Archer, Nano Today, 2015, 10, 315-338.

8 X. Ji, K. T. Lee and L. F. Nazar, Nat. Mater., 2009, 8, 500-506. 9 J. Liu, T. Yang, D.-W. Wang, G. Q. Lu, D. Zhao and S. Z. Qiao, Nat. Commun., 2013, 4, 2798.

10 R. Fang, S. Zhao, P. Hou, M. Cheng, S. Wang, H.-M. Cheng, C. Liu and F. Li, Adv. Mater., 2016, 28, 3374-3382.

11 X.-B. Cheng, J.-Q. Huang, Q. Zhang, H.-J. Peng, M.-Q. Zhao and F. Wei, Nano Energy, 2014, 4, 65-72.

12 L. Fei, X. Li, W. Bi, Z. Zhuo, W. Wei, L. Sun, W. Lu, X. Wu, K. Xie, C. Wu, H. L. W. Chan and Y. Wang, Adv. Mater., 2015, 27, 5936-5942.

13 J. Zhu, D. Yang, Z. Yin, Q. Yan and H. Zhang, Small, 2014, 10, 3480-3498.

14 M. Yu, R. Li, M. Wu and G. Shi, Energy Storage Materials, 2015, 1, 51-73.

15 R. Chen, T. Zhao, J. Lu, F. Wu, L. Li, J. Chen, G. Tan, Y. Ye and K. Amine, Nano Lett., 2013, 13, 4642-4649. 
16 L. Ji, M. Rao, H. Zheng, L. Zhang, Y. Li, W. Duan, J. Guo, E. J. Cairns and Y. Zhang, J. Am. Chem. Soc., 2011, 133, 18522-18525.

17 C. Zu and A. Manthiram, Adv. Energy Mater., 2013, 3, 10081012.

18 S. Pei and H.-M. Cheng, Carbon, 2012, 50, 3210-3228.

19 R. K. Singh, R. Kumar and D. P. Singh, RSC Adv., 2016, 6, 64993-65011.

20 K. C. Wasalathilake, G. A. Ayoko and C. Yan, in Recent Advances in Graphene Research, InTech, 2016, DOI: 10.5772/ 63554.

21 X. Huang, B. Sun, K. Li, S. Chen and G. Wang, J. Mater. Chem. A, 2013, 1, 13484-13489.

22 K. H. Kim, Y.-S. Jun, J. A. Gerbec, K. A. See, G. D. Stucky and H.-T. Jung, Carbon, 2014, 69, 543-551.

23 G. Zhou, L.-C. Yin, D.-W. Wang, L. Li, S. Pei, I. R. Gentle, F. Li and H.-M. Cheng, ACS Nano, 2013, 7, 5367-5375.

24 W. Ahn, M. H. Seo, Y.-S. Jun, D. U. Lee, F. M. Hassan, X. Wang, A. Yu and Z. Chen, ACS Appl. Mater. Interfaces, 2016, 8, 1984-1991.

25 Z. Li, X. Li, Y. Liao, X. Li and W. Li, J. Power Sources, 2016, 334, 23-30.

26 K. Yang, Q. Gao, Y. Tan, W. Tian, L. Zhu and C. Yang, Microporous Mesoporous Mater., 2015, 204, 235-241.

27 X. Gu, Y. Wang, C. Lai, J. Qiu, S. Li, Y. Hou, W. Martens, N. Mahmood and S. Zhang, Nano Res., 2015, 8, 129-139.

28 M. Vijayakumar, N. Govind, E. Walter, S. D. Burton, A. Shukla, A. Devaraj, J. Xiao, J. Liu, C. Wang, A. Karim and S. Thevuthasan, Phys. Chem. Chem. Phys., 2014, 16, 10923-10932.

29 L. Wang, T. Zhang, S. Yang, F. Cheng, J. Liang and J. Chen, J. Energy Chem., 2013, 22, 72-77.

30 S. P. Jand, Y. Chen and P. Kaghazchi, J. Power Sources, 2016, 308, 166-171.

31 T.-Z. Hou, X. Chen, H.-J. Peng, J.-Q. Huang, B.-Q. Li, Q. Zhang and B. Li, Small, 2016, 12, 3283-3291.

32 H. Ting-Zheng, P. Hong-Jie, H. Jia-Qi, Z. Qiang and L. Bo, 2D Mater., 2015, 2, 014011.

33 Y.-X. Yin, S. Xin, Y.-G. Guo and L.-J. Wan, Angew. Chem., Int. Ed., 2013, 52, 13186-13200.

34 H. Liao, H. Ding, B. Li, X. Ai and C. Wang, J. Mater. Chem. A, 2014, 2, 8854-8858.
35 D. R. Dreyer, S. Park, C. W. Bielawski and R. S. Ruoff, Chem. Soc. Rev., 2010, 39, 228-240.

36 A. S. Dobrota, I. A. Pasti, S. V. Mentus and N. V. Skorodumova, Phys. Chem. Chem. Phys., 2016, 18, 6580-6586.

37 B. Delley, J. Chem. Phys., 1990, 92, 508-517.

38 B. Delley, J. Chem. Phys., 2000, 113, 7756-7764.

39 J. P. Perdew, K. Burke and M. Ernzerhof, Phys. Rev. Lett., 1996, 77, 3865-3868.

40 E. R. McNellis, J. Meyer and K. Reuter, Phys. Rev. B: Condens. Matter Mater. Phys., 2009, 80, 205414.

41 H. J. Monkhorst and J. D. Pack, Phys. Rev. B: Solid State, 1976, 13, 5188-5192.

42 B. Wang, S. M. Alhassan and S. T. Pantelides, Phys. Rev. Appl., 2014, 2, 034004.

43 Z. Ji, B. Han, Q. Li, C. Zhou, Q. Gao, K. Xia and J. Wu, J. Phys. Chem. C, 2015, 119, 20495-20502.

44 M. Hagen, P. Schiffels, M. Hammer, S. Dörfler, J. Tübke, M. J. Hoffmann, H. Althues and S. Kaskel, J. Electrochem. Soc., 2013, 160, A1205-A1214.

45 Q. Zhang, Y. Wang, Z. W. Seh, Z. Fu, R. Zhang and Y. Cui, Nano Lett., 2015, 15, 3780-3786.

46 J. Zhao, Y. Yang, R. S. Katiyar and Z. Chen, J. Mater. Chem. A, 2016, 4, 6124-6130.

47 S. Li, T. Mou, G. Ren, J. Warzywoda, B. Wang and Z. Fan, ACS Energy Lett., 2016, 1, 481-489.

48 N. G. Szwacki and T. Szwacka, Basic Elements of Crystallography, Pan Stanford, 2nd edn, 2016.

49 L. Fan, H. L. Zhuang, K. Zhang, V. R. Cooper, Q. Li and Y. Lu, Adv. Sci., 2016, 3, 1600175.

50 Y. Zhong, K. R. Yang, W. Liu, P. He, V. Batista and H. Wang, J. Phys. Chem. C, 2017, 121, 14222-14227.

51 X. Chen, H.-J. Peng, R. Zhang, T.-Z. Hou, J.-Q. Huang, B. Li and Q. Zhang, ACS Energy Lett., 2017, 2, 795-801.

52 Y. Zhao and J. Zhao, Appl. Surf. Sci., 2017, 412, 591-598.

53 D. W. Boukhvalov and M. I. Katsnelson, J. Am. Chem. Soc., 2008, 130, 10697-10701.

54 M. Barhoumi, D. Rocca, M. Said and S. Lebègue, J. Chem. Phys., 2017, 146, 044705.

55 S. Stankovich, D. A. Dikin, R. D. Piner, K. A. Kohlhaas, A. Kleinhammes, Y. Jia, Y. Wu, S. T. Nguyen and R. S. Ruoff, Carbon, 2007, 45, 1558-1565. 